\title{
IDEALS ON GENERALIZED TOPOLOGICAL SPACES
}

\author{
FAHAD ALSHARARI* \\ Department of Mathematics, College of Science and Human Studies, Hotat Sudair, Majmaah University, \\ Majmaah 11952, Saudi Arabia \\ *Corresponding author: f.alsharari@mu.edu.sa
}

\begin{abstract}
In this paper, we define the g-closure operator and investigate some of its crucial properties. We also introduce and study the concept of $\psi_{\mathrm{g}}$-classes and generalized compatibly of generalized topology with ideal. This work is generalization of [4].
\end{abstract}

\section{INTRODUCTION}

The idea of "idealizing" of a topological space can be found in some classical texts of Kuratowski ( [14], [15]) and Vaidyanathaswamy [18]. Some early applications of ideal topological spaces can be found in various branches of mathematics, like a generalization of Cantor-Bendixson theorem by Freud [10], or in measure theory by Scheinberg [17]. In 1990 Jankovic̀ and Hamlett [13] wrote a paper in which they, among their results, included many other results in this area using modern notation, and logically and systematically arranging them. This paper rekindled the interest in this topic, resulting in many generalizations of the ideal topological space and many generalizations of the notion of open sets, like in papers of Jafari and Rajesh [11], and Manoharan and Thangavelu [16].

In 1966 Velicko [19] introduced the notions of $\theta$-open and $\theta$-closed sets, and also a $\theta$-closure, examining $H$-closed spaces in terms of an arbitrary filterbase. A space $X$ is called $H$-closed if every open cover of $X$ has a finite subfamily whose closures cover $X$. It turned out that $\theta$-open sets completely correspond to the

Received 2019-11-22; accepted 2020-01-13; published 2020-03-02.

2010 Mathematics Subject Classification. 54A05.

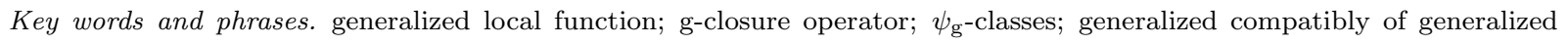
topology with ideal.

(C) 2020 Authors retain the copyrights of their papers, and all open access articles are distributed under the terms of the Creative Commons Attribution License. 
already known notion of $\theta$-continuity, introduced in 1943 by Fomin [9]. In 1975 Dickman and Porter [8] continued the study of $H$-closed spaces using $\theta$-closed sets proving that an $H$-closed space is not a countable union of nowhere dense $\theta$-closed sets. In 1980, Jankovic̀ [12] proved that a space is Hausdorff if and only if every compact set is $\theta$-closed. Recent applications of $\theta$-open sets can be found in the paper of Caldas, Jafari and Latif [6], and in the paper of Cammaroto, Catalioto, Pansera and Tsaban [7].

In [5], Al-Omari and Noiri introduced the local closure function as a generalization of the $\theta$-closure and the local function in an ideal topological space. They proved some basic properties for the local closure function, and also introduced two new topologies obtained from the original one using the local closure function. Afterwards, many properties in topological spaces have been explored by various researchers $([20],[21],[22],[23],[24],[25])$

Ideal topological space is a beautiful mixture of topology and geometry. A generalized topology (briefly, $G T)[1], \mu$ on a nonempty set $X$ is a collection of subsets of $X$ such that $\phi \in \mu$ and $\mu$ is closed under arbitrary unions. Elements of $\mu$ will be called $\mu$-open sets, and a subset $\mathcal{A}$ of $(X, \mu)$ will be called $\mu$-closed if $(X \backslash \mathcal{A})$ is $\mu$-open. The pair $(X, \mu)$ will be called a generalized topological space (briefly, GTS) ( [2], [3]). By a space $X$ or $(X, \mu)$, we will always mean a GTS. Clearly, every topological space is a GTS.

If $U$ is a subset of a space $(X, \mu)$, then the $\mu$-closure $\mathrm{Cl}_{\mu}(U)$ of $\mu$ is the intersection of all $\mu$-closed sets containing $U$ and the $\mu$-interior $\operatorname{int}_{\mu}(U)$ of $U$ is the union of all $\mu$-open sets contained in $\mu$ [2].

Let $(X, \tau)$ be a topological space, for each $U \in P(X)$ and $x \in X$. Then, $U$ is called generalized open neighborhood of $x$ if $x \in U$ with $U$ is $\mu$-open set. That is $N_{\mathrm{g}}(x)=\{U$ is $\mu$-open: $x \in U\}$.

\section{Ideal Generalized Local Function}

In this section we introduce and study the concept of ideal generalized topological spaces. We also investigate some of its properties.

Definition 2.1. An ideal generalized topological space is a generalized topological space $(X, \mu)$ with an ideal $I$ on $X$ and is denoted by $(X, \mu, I)$. For a subset $\mathcal{A} \subset X, \mathcal{A}_{g}(I, \mu)=\left\{x \in X: U \cap \mathcal{A} \notin I\right.$ for each $\left.U \in N_{\mathrm{g}}(x)\right\}$.

Clearly, every ideal topological space is ideal generalized topological space.

Lemma 2.1. For $\mathcal{A} \subseteq(X, \mu, I)$ we have, $C l_{\mu}\left(\mathcal{A}_{g}\right)=\mathcal{A}_{g}$

Proof. One implication is immediate. Conversely, we show that $C l_{\mu}\left(\mathcal{A}_{g}\right) \subseteq \mathcal{A}_{g}$.

Let $x \in C l_{\mu}\left(\mathcal{A}_{g}\right)$. Then for each $\mu$-open set $G$ containing $x, G \cap \mathcal{A}_{g} \neq \phi$. Thus, $x \in \mathcal{A}_{g}(I, \mu)$ which implies, for each generalized open neighborhood $U$ of $x, U \cap \mathcal{A} \notin I$. Therefore, $(G \cup U) \cap \mathcal{A} \notin I$, implies $x \in \mathcal{A}_{g}(I, \mu)$. Hence, $C l_{\mu}\left(\mathcal{A}_{g}\right) \subseteq \mathcal{A}_{g}$. which completes the proof 
Corollary 2.1. Let $(X, \mu, I)$ be an ideal generalized topological space. Then, $\mathcal{A}_{g}(\phi, \mu)=C l_{\mu}(\mathcal{A})$.

Proof. Follows from Lemma 2.1

Theorem 2.1. Let $(X, \mu, I)$ be an ideal generalized topological space and $\mathcal{A}, \mathcal{B} \subseteq X$. Then:

(1) $\mathcal{A}_{g}$ is $\mu$-closed set,

(2) If $I_{1} \subseteq I_{2}$, then $\mathcal{A}_{g}\left(I_{1}\right) \supseteq \mathcal{A}_{g}\left(I_{2}\right)$,

(3) If $\mathcal{A} \subseteq \mathcal{B}$, then $\mathcal{A}_{g} \subseteq \mathcal{B}_{g}$,

(4) $\mathcal{A}_{g} \subseteq C l_{\mu}(\mathcal{A})$,

(5) $\left(\mathcal{A}_{g}\right)_{g} \subseteq \mathcal{A}_{g}$

(6) $\mathcal{A}_{g} \cup \mathcal{B}_{g}=(\mathcal{A} \cup \mathcal{B})_{g}$,

(7) $\mathcal{A}_{g}-\mathcal{B}_{g}=(\mathcal{A}-\mathcal{B})_{g}$,

(8) If $\mathcal{B} \in I$, then $(\mathcal{A} \cup \mathcal{B})_{g}=(\mathcal{A}-\mathcal{B})_{g}=\mathcal{A}_{g}$,

(9) If $\mathcal{B} \in I$, then $(X-\mathcal{B})_{g}=X_{g}$,

(10) If $U \in \mu$, then $\left(U \cap \mathcal{A}_{g}\right) \subseteq(U \cap \mathcal{A})_{g}$.

Proof. (1) If $x \notin \mathcal{A}_{g}$, then for some $\mu$-open set $U$, we have $x \in U$ and $U \cap \mathcal{A} \in I$. This implies $U \subseteq X-\mathcal{A}_{g}$, which means that $X-\mathcal{A}_{g}$ is $\mu$-open set. Thus, $\mathcal{A}_{g}$ is $\mu$-closed set.

(2) It is clear.

(3) Let $x \notin \mathcal{B}_{g}$. Then, there exists $U \in N_{g}(x)$ containing $x$ such that $U \cap \mathcal{B} \in I$. This implies $U \cap \mathcal{A} \subseteq U \cap \mathcal{B} \in I$. Hence, $x \notin \mathcal{A}_{g}$. Thus, $\mathcal{A}_{g} \subseteq \mathcal{B}_{g}$.

(4) follows directly from (1). 
(5) Since $\mathcal{A}_{g} \subseteq C l \mu(\mathcal{A}),\left(\mathcal{A}_{g}\right)_{g} \subseteq C l_{\mu}\left(\mathcal{A}_{g}\right)$. By $(1),\left(\mathcal{A}_{g}\right)_{g} \subseteq C l_{\mu}\left(\mathcal{A}_{g}\right)=\mathcal{A}_{g}$.

(6) One implication is immediate from (2), that is $\left(\mathcal{A}_{g} \cup \mathcal{B}_{g}\right) \subset(\mathcal{A} \cup \mathcal{B}) g$. To prove the reveres inclusion. Let $x \notin\left(\mathcal{A}_{g} \cup \mathcal{B}_{g}\right)$, then $x \notin \mathcal{A}_{g}$ or $x \notin \mathcal{B}_{g}$. Then there exists $U 1 \in N_{g}(x)$ and $U_{2} \in N_{g}(x)$ such that $U_{1} \cap \mathcal{A} \in I$ and $U_{2} \cap \mathcal{B} \in I$. Since $I$ is hereditary and additive, then $\left(U_{1} \cap U_{2}\right) \cap(\mathcal{A} \cup \mathcal{B}) \in I$. Thus, $x \notin(\mathcal{A} \cup \mathcal{B})_{g}$. Hence, $\left(\mathcal{A}_{g} \cup \mathcal{B}_{g}\right) \supset(\mathcal{A} \cup \mathcal{B})_{g}$.

(7) Since $\mathcal{A}=(\mathcal{A} \cap \mathcal{B}) \cup(\mathcal{A}-\mathcal{B})$ for any $\mathcal{A}, \mathcal{B} \subseteq X$. Then by (2), we have

$$
\mathcal{A}_{g}-\mathcal{B}_{g}=[(\mathcal{A} \cap \mathcal{B}) \cup(\mathcal{A}-\mathcal{B})]_{g}-\mathcal{B}_{g}=(\mathcal{A}-\mathcal{B})_{g}
$$

(8) By using (6) and the fact that, if $\mathcal{B} \in I$, then $\mathcal{B}_{g}=\phi$.

(9) Obvious.

(10) Let $x \in U \cap \mathcal{A}_{g}$. Then $x \in U$ and $x \in \mathcal{A}_{g}$. This implies that there exists $G \in N_{g}(x)$ such that $G \cap \mathcal{A} \in I$. Since, $x \in U \cap G, U \cap(G \cap \mathcal{A}) \in I$. Hence, $x \in(U \cap \mathcal{A})_{g}$.

Theorem 2.2. Let $(X, \mu, I)$ be an ideal generalized topological space and $\left\{\mathcal{A}_{i}\right\}_{i \in J}$ be a family of subsets of X. Then:

(1) $\left(\bigcup\left(\mathcal{A}_{i}\right)_{g}: i \in J\right) \subseteq\left(\bigcup \mathcal{A}_{i}: i \in J\right)_{g}$,

(2) $\left(\bigcap \mathcal{A}_{i}: i \in J\right)_{g} \subseteq\left(\bigcap\left(\mathcal{A}_{i}\right)_{g}: i \in J\right)$,

Proof. (1) Since $\mathcal{A}_{i} \subseteq \cup \mathcal{A}_{i}$, for each $i \in J$, by Theorem 2.1(2), we have $\left(\mathcal{A}_{i}\right)_{g} \subseteq\left(\bigcup \mathcal{A}_{i}\right)_{g}$, for each $i \in J$. This implies

$$
\left(\bigcup\left(\mathcal{A}_{i}\right)_{g}: i \in J\right) \subseteq\left(\bigcup \mathcal{A}_{i}: i \in J\right)_{g}
$$

(2) Since $\bigcap \mathcal{A}_{i} \subseteq \mathcal{A}_{i},\left(\bigcap \mathcal{A}_{i}\right)_{g} \subseteq\left(\mathcal{A}_{i}\right)_{g}$, for each $i \in J$. Thus,

$$
\left(\bigcap \mathcal{A}_{i}: i \in J\right)_{g} \subseteq\left(\bigcap\left(\mathcal{A}_{i}\right)_{g}: i \in J\right)
$$

Now, we define the g-closure operator, denoted by $C l_{\mu}^{\star}$ for a generalized topology $\mu^{\star}(I)$ finer than $\tau$ as follows: $C l_{\mu}^{\star}(\mathcal{A})=\mathcal{A} \cup \mathcal{A}_{g}$ for every $\mathcal{A} \subseteq X$. We will occasionally write $\mathcal{A}_{g}$ or $\mathcal{A}_{g}(I)$ for $\mathcal{A}_{g}(\mu, I)$ and it will cause no ambiguity.

We will denote by $i n t_{\mu}^{\star}(\mathcal{A})$ and $C l_{\mu}^{\star}(\mathcal{A})$ the interior and closure of $\mathcal{A} \subseteq(X, \mu, I)$, respectively, with respect to $\mu^{\star}$. 
Theorem 2.3. The class $\beta(\mu, I)=\{U-\mathcal{E}: U \in \mu, \mathcal{E} \in I\}$ is a base for a generalized topology.

Proof. For every $\beta_{1}, \beta_{2} \in \beta$, we have $\beta_{1}=U_{1}-\mathcal{E}_{1}$ and $\beta_{2}=U_{2}-\mathcal{E}_{2}$ where $U_{1}, U_{2} \in \mu$ and $\mathcal{E}_{1}, \mathcal{E}_{2} \in I$. Then

$$
\begin{gathered}
\beta_{1} \cap \beta 2=\left(U_{1}-\mathcal{E}_{1}\right) \cap\left(U_{2}-\mathcal{E}_{2}\right)=\left(U_{1} \cap X-\mathcal{E}_{1}\right) \cap\left(U_{2} \cap X-\mathcal{E}_{2}\right) \\
=\left(U_{1} \cap U_{2}\right) \cap\left(X-\mathcal{E}_{1} \cap X-\mathcal{E}_{2}\right)=\left(U_{1} \cap U_{2}\right) \cap X-\left(\mathcal{E}_{1} \cup \mathcal{E}_{2}\right) \\
=\left(U_{1} \cap U_{2}\right)-\left(\mathcal{E}_{1} \cup \mathcal{E}_{2}\right) \in \beta
\end{gathered}
$$

The generalized topology which have $\beta(\mu, I)$ as a base is called $\star$-generalized topology and is denoted by $\mu^{\star}$.

Theorem 2.4. Let $(X, \mu, I)$ be an ideal generalized topological space. Then for each $\mathcal{A}, \mathcal{B} \in(X, \mu, I)$ we have:

(1) If $\mathcal{A} \subseteq \mathcal{B}$, then $C l_{g}^{\star}(\mathcal{A}) \subseteq C l_{g}^{\star}(\mathcal{B})$.

(2) $C l_{\mu}^{\star}\left(C l_{\mu}^{\star}(\mathcal{A})\right) \subseteq C l_{\mu}^{\star}(\mathcal{A})$.

(3) $C l_{\mu}^{\star}(\mathcal{A} \cup \mathcal{B})=C l_{\mu}^{\star}(\mathcal{A}) \cup C l_{\mu}^{\star}(\mathcal{B})$.

(4) $\mathcal{A} \subseteq C l_{\mu}^{\star}(\mathcal{A}) \subseteq C l_{\mu}(\mathcal{A})$

(5) If $I=\phi$, then, $\mathcal{A}_{g}=C l_{\mu}(\mathcal{A}) \subseteq C l(\mathcal{A})$.

(6) If $I=P_{X}$, then, $\mathcal{A}_{g}=\phi$ and $\mathcal{A}=C l_{\mu}(\mathcal{A})$.

Proof. We shall verify only the statements (2) and the remainder of this theorem can be proved similarly

(2) Form Theorem 2.1(5) we have

$$
\begin{gathered}
C l_{\mu}^{\star}\left(C l_{\mu}^{\star}(\mathcal{A})\right)=C l_{\mu}^{\star}(\mathcal{A}) \cup\left(C l_{\mu}^{\star}(\mathcal{A})_{\mu}=\left(\mathcal{A} \cup \mathcal{A}_{\mu}\right) \cup\left(\mathcal{A} \cup \mathcal{A}_{\mu}\right)_{\mu}\right. \\
=\left(\mathcal{A} \cup \mathcal{A}_{\mu}\right)=C l_{\mu}^{\star}(\mathcal{A}) .
\end{gathered}
$$

Theorem 2.5. If $I_{1}$ and $I_{2}$ are two ideals on $(X, \mu)$ such that $I_{1} \subseteq I_{2}$, then:

(1) $\mathcal{A}_{g}\left(I_{1}\right) \supseteq \mathcal{A}_{g}\left(I_{2}\right)$. 
(2) $\mu^{\star}\left(I_{1}\right) \subseteq \mu^{\star}\left(I_{2}\right)$.

Proof. (1) Since $\mathcal{A}$ is a generalized open local function of $I_{1}$ at $x$, it must also be a generalized open local function of $I_{2}$ at $x$ (since every $I_{1}$ is $\left.I_{2}\right)$. Hence, $\mathcal{A}_{g}\left(I_{1}\right) \supseteq \mathcal{A}_{g}\left(I_{2}\right)$.

(2) Since $I_{1} \subseteq I_{2}$ and by Theorem $2.1(2), \mathcal{A}_{\mu}\left(I_{1}\right) \supseteq \mathcal{A}_{g}\left(I_{2}\right)$. This implies that $C l_{\mu}^{\star}(\mathcal{A})\left(I_{2}, \mu\right) \subseteq$ $\left.C l_{\mu}^{\star}(\mathcal{A})\left(I_{1}, \mu\right)\right)$. Therefore, $\mu^{\star}\left(I_{1}\right) \subseteq \mu^{\star}\left(I_{2}\right)$.

Theorem 2.6. If $(X, \mu, I)$ is an ideal generalized topological space and $\mathcal{A} \subseteq X$. Then $\mathcal{A}_{g}-\left(\mathcal{A}_{g}\right)_{g} \subseteq\left(\mathcal{A}-\mathcal{A}_{g}\right)_{g}$.

Proof. Let $x \in \mathcal{A}_{g}-\left(\mathcal{A}_{g}\right)_{g}$. Then $x \in \mathcal{A}_{g} \cap\left(X-\left(\mathcal{A}_{g}\right)_{g}\right)$. Thus, $x \in \mathcal{A}_{g}$ that is, there exists $U \in N_{g}(x)$ such that $U \cap \mathcal{A} \notin I$. Hence, for each $U \in N_{g}(x), U \cap\left(\mathcal{A}-\mathcal{A}_{g}\right) \notin I$, which implies $x \in\left(\mathcal{A}-\mathcal{A}_{g}\right)$ and this completes the proof.

Theorem 2.7. Let $(X, \mu)$ be a generalized topological space with $I_{1}$ and $I_{2}$ two ideals on $X$ and $\mathcal{A} \subseteq X$.

Then:

(1) $\mathcal{A}_{g}\left(I_{1} \cap I_{2}, \mu\right)=\mathcal{A}_{g}\left(I_{1}, \mu\right) \cup \mathcal{A}_{g}\left(I_{2}, \mu\right)$.

(2) $\mathcal{A}_{g}\left(I_{1} \cup I_{2}, \mu\right)=\mathcal{A}_{g}\left(I_{1}, \mu^{\star}\left(I_{2}\right)\right) \cap \mathcal{A}_{g}\left(I_{2}, \mu^{\star}\left(I_{1}\right)\right)$.

Proof. (1) Since $I_{1} \cap I_{2} \subseteq I_{1}$, then from Theorem 2.1 (2) we have $\mathcal{A}_{g}\left(I_{1}, \mu\right) \subseteq \mathcal{A}_{g}\left(I_{1} \cap I_{2}, \mu\right)$. Similarly, $\mathcal{A}_{g}\left(I_{2}, \mu\right) \subseteq \mathcal{A}_{g}\left(I_{1} \cap I_{2}, \mu\right)$. Hence,

$$
\mathcal{A}_{g}\left(I_{1}, \mu\right) \cup \mathcal{A}_{g}\left(I_{2}, \mu\right) \subseteq \mathcal{A}_{g}\left(I_{1} \cap I_{2}, \mu\right)
$$

To prove the reverse inclusion, let $x \notin \mathcal{A}_{g}\left(I_{1}, \mu\right) \cup \mathcal{A}_{g}\left(I_{2}, \mu\right)$, then $x \notin \mathcal{A}_{g}\left(I_{1}, \mu\right)$ and $x \notin \mathcal{A}_{g}\left(I_{2}, \mu\right)$, implies that there exists $U_{1} \in N g(x)$ such that $U_{1} \cap \mathcal{A} \in I_{1}$. Again, $x \notin \mathcal{A}_{g}\left(I_{2}, \mu\right)$, implies there exists $U_{2} \in N g(x)$ such that $U_{2} \cap \mathcal{A} \in I_{2} .$. Therefore, $\left(U_{1} \cap U 2\right) \cap \mathcal{A} \in\left(I_{1} \cap I_{2}\right)$. Hence, $x \notin \mathcal{A}_{g}\left(I_{1} \cap I_{2}, \mu\right)$, implies that

$$
\mathcal{A}_{g}\left(I_{1} \cap I_{2}, \mu\right) \subseteq \mathcal{A}_{g}\left(I_{1}, \mu\right) \cup \mathcal{A}_{g}\left(I_{2}, \mu\right)
$$

Therefore, equations (2.1) and (2.2) establish the result.

(2) Assume that $x \notin \mathcal{A}_{g}\left(I_{1} \cup I_{2}, \mu\right)$, then there exists $U \in N g(x)$ such that $U \cap \mathcal{A} \in I_{1} \cup I 2$. Let $\mathcal{E} \in I_{1}$ and $\mathcal{H} \in I_{2}$ such that $U \cap \mathcal{A}=\mathcal{E} \cup \mathcal{H}$, because of the heredity of $I$, we may assume $\mathcal{E} \cup \mathcal{H}=\phi$. Thus we have $U \cap \mathcal{A}-\mathcal{E}=\mathcal{H}$ and $U \cap \mathcal{A}-\mathcal{H}=\mathcal{E}$. Thus, $(U-\mathcal{E}) \cap \mathcal{A}=\mathcal{H} \in I_{1}$ and $(U-\mathcal{H}) \cap \mathcal{A}=\mathcal{E} \in I_{2}$. Therefore, $x \notin \mathcal{A}_{g}\left(I_{2}, \mu^{\star}\left(I_{1}\right)\right)$ or $x \notin \mathcal{A}_{g}\left(I_{1}, \mu^{\star}\left(I_{2}\right)\right)$ and hence,

$$
\mathcal{A}_{g}\left(I_{1}, \mu^{\star}\left(I_{2}\right)\right) \cap \mathcal{A}_{g}\left(I_{2}, \mu^{\star}\left(I_{1}\right)\right) \subseteq \mathcal{A}_{g}\left(I_{1} \cup I_{2}, \mu\right) .
$$


Now assume that $x \notin \mathcal{A}_{g}\left(I_{1}, \mu^{\star}\left(I_{2}\right)\right)$. This implies that there exist $U \in N_{g}(x)$ and $\mathcal{H} \in I_{2}$ such that $(U-\mathcal{H}) \cap \mathcal{A} \in I_{1}$. We may assume, because of the heredity of $I_{2}$, that $\mathcal{H} \subseteq \mathcal{A}$. Put $\mathcal{E}=(U-\mathcal{H}) \cap \mathcal{A}$ and we have $U \cap \mathcal{A}=\mathcal{E} \cup \mathcal{H} \in I_{1} \cup I_{2}$. Thus, $x \notin \mathcal{A}_{g}\left(I_{1} \cup I_{2}, \mu\right)$. Thus we have shown that $\mathcal{A}_{g}\left(I_{1} \cup I_{2}, \mu\right) \subseteq \mathcal{A}_{g}\left(I_{1}, \mu^{\star}\left(I_{2}\right)\right)$.

Similarly, we have that $\mathcal{A}_{g}\left(I_{1} \cup I_{2}, \mu\right) \subseteq \mathcal{A}_{g}\left(I_{2}, \mu^{\star}\left(I_{1}\right)\right)$. Therefore,

$$
\mathcal{A}_{g}\left(I_{1} \cup I_{2}, \mu\right) \subseteq \mathcal{A}_{g}\left(I_{1}, \mu^{\star}\left(I_{2}\right)\right) \cap \mathcal{A}_{g}\left(I_{2}, \mu^{\star}\left(I_{1}\right)\right)
$$

From (2.3) and (2.4) the result is establised.

Remark 2.1. Put $I_{1}=I_{2}$ in the above theorem, the following Corollary answers about the relationship between $\mu^{\star}$ and $\left[\mu^{\star}\right]^{\star}$.

Corollary 2.2. Let $(X, \mu)$ be a generalized topological space with $I$ an ideal on $X$ and $\mathcal{A} \subseteq X$. Then:

(1) $\mathcal{A}_{g}\left(I_{1}, \mu\right)=\mathcal{A}_{g}\left(I_{1}, \mu^{\star}\right)$

(2) $\mu^{\star}=\left[\mu^{\star}\right]^{\star}$

Definition 2.2. A subset $\mathcal{A}$ of the space $(X, \mu, I)$ is said to be $\mu$-closed set iff $\mathcal{A}_{g} \subseteq \mathcal{A}$. Equivalently, $\mathcal{A}$ is said to be $\mu^{\star}$-closed iff $C l_{\mu}^{\star}(\mathcal{A})=\mathcal{A}$.

Theorem 2.8. The following statements are equivalent for a subset $\mathcal{A}$ of a space $(X, \mu, I)$.

(1) $\mathcal{A} \in \mu^{\star}$.

(2) $\mathcal{A}$ is $\mu^{\star}$-closed set.

(3) $(X-\mathcal{A})_{g} \subseteq(X-\mathcal{A})$.

(4) $\mathcal{A} \subseteq(X-\mathcal{A})_{g}$.

Proof. It is clear.

\section{3. $\Psi_{\mathrm{g}}$-Classes}

Definition 3.1. If $(X, \mu, I)$ is ideal generalized topological spaces, we define an operator $\Psi_{\mathrm{g}}(\mu, I): P_{X} \rightarrow \mu$ as follows: for every $\mathcal{A} \subseteq X, \Psi_{\mathrm{g}}(\mu, I)(\mathcal{A})=\left\{x\right.$ : there exists $U \in N_{g}(x)$ such that $\left.U-\mathcal{A} \in I\right\}$. Equivalently, $\Psi_{\mathrm{g}}(\mu, I)(\mathcal{A})=X-(X-\mathcal{A})_{g}$ for each $\mathcal{A} \subseteq X$. We denote $\Psi_{\mathrm{g}}(\mu, I)$ by $\Psi_{\mathrm{g}}$ when no ambiguity.

Lemma 3.1. For $\mathcal{A} \subseteq(X, \mu, I)$ we have:

(1) If $I=\phi$, then $\Psi_{\mathrm{g}}(\mathcal{A})=\operatorname{int}_{\mu}(\mathcal{A})$.

(2) If $I=P_{X}$, then $\Psi_{\mathrm{g}}(\mathcal{A})=X$. 
Proof. Obvious.

Theorem 3.1. For $\mathcal{A} \subseteq(X, \mu, I)$ we have:

:

(1) $\Psi_{\mathrm{g}}(\mathcal{A})=\cup\{U \in \mu: U-\mathcal{A} \in I\}$.

(2) If $U \in \mu$, then $\Psi_{\mathrm{g}}(U)=\cup\{\mathcal{M} \in \mu:(\mathcal{M}-U) \cup(U-\mathcal{M}) \in I\}$.

Proof. (1) Follows immediately form Definition 3.1.

(2) ) If we denote $\cup\{\mathcal{M} \in \mu:(\mathcal{M}-\mathcal{A}) \cup(U-\mathcal{M}) \in I\}$ by $\Psi_{\mathrm{g}^{\prime}}(\mathcal{A})_{1}$. By heredity of $I$ we have $\{\mathcal{M} \in \mu:(\mathcal{M}-\mathcal{A}) \cup(U-\mathcal{M}) \in I\} \subseteq\{\mathcal{M} \in \mu:(\mathcal{M}-\mathcal{A}) \in I\}$ and hence by (1) we have for every $\mathcal{A} \subseteq X$

$$
\Psi_{\mathrm{g}^{\prime}}(\mathcal{A}) \subseteq \Psi_{\mathrm{g}}(\mathcal{A})
$$

Now assume that $U \in \mu$ and $x \in \Psi_{\mathrm{g}}(U)$. Then there exists $\mathcal{M} \in \mu$ such that $x \in(\mathcal{M}-U) \in I$. Let $\mathcal{N}=\mathcal{M} \cup U$. Then, $\mathcal{N} \in \mu$ and $x \in(\mathcal{N}-U) \cup(U-\mathcal{N})=(\mathrm{M}-U) \cup \phi=(\mathcal{M}-U) \in I$. Thus, $x \in \Psi_{\mathrm{g}^{\prime}}(U)$. Hence

$$
\Psi_{\mathrm{g}}(U) \subseteq \Psi_{\mathrm{g}^{\prime}}(U)
$$

From (3.1) and (3.2) we have $\Psi_{\mathrm{g}}(U)=\Psi_{\mathrm{g}^{\prime}}(U)$, for every $U \in \mu$.

Theorem 3.2. Let $\mathcal{A} \subseteq(X, \mu, I)$ be a generalized topological space.

(1) If $\mathcal{A} \subseteq X$, then $\Psi_{\mathrm{g}}(\mathcal{A})$ is $\mu$-open set.

(2) If $\mathcal{A} \subseteq \mathcal{B}$, then $\Psi_{\mathrm{g}}(\mathcal{A}) \subseteq \Psi_{\mathrm{g}}(\mathcal{B})$.

(3) If $\mathcal{A}, \mathcal{B} \in P_{X}$, then $\Psi_{\mathrm{g}}(\mathcal{A} \cap \mathcal{B})=\Psi_{\mathrm{g}}(\mathcal{A}) \cap \Psi_{\mathrm{g}}(\mathcal{B})$.

(4) If $U \in \mu$, then $U \in \Psi_{\mathrm{g}}(\mathcal{U})$.

(5) If $\mathcal{A} \subseteq X$, then $\Psi_{\mathrm{g}}(\mathcal{A}) \subseteq \Psi_{\mathrm{g}}\left(\Psi_{\mathrm{g}}(\mathcal{A})\right)$.

(6) If $\mathcal{A} \subseteq X$, then $\Psi_{\mathrm{g}}(\mathcal{A})=\Psi_{\mathrm{g}}\left(\Psi_{\mathrm{g}}(\mathcal{A})\right)$ iff $(X-\mathcal{A})_{g}=\left((X-\mathcal{A})_{g}\right)_{g}$.

(7) If $\mathcal{A} \subseteq I$, then $\Psi_{\mathrm{g}}(\mathcal{A})=\left(X-\mathcal{A}_{g}\right)$.

(8) If $\mathcal{A} \subseteq X$ and $\mathcal{E} \subseteq I$, then $\Psi_{\mathrm{g}}(\mathcal{A}-\mathcal{E})=\Psi_{\mathrm{g}}(\mathcal{A})$.

(9) If $\mathcal{A} \subseteq X$ and $\mathcal{E} \subseteq I$, then $\Psi_{\mathrm{g}}(\mathcal{A} \cup \mathcal{E})=\Psi_{\mathrm{g}}(\mathcal{A})$.

(10) If $(\mathcal{A}-\mathcal{B}) \cup(\mathcal{B}-\mathcal{A}) \in I$, then $\Psi_{\mathrm{g}}(\mathcal{A})=\Psi_{\mathrm{g}}(\mathcal{B})$.

Proof. (1) Follows from Theorem 3.2(1). 
(2) Since $\Psi_{\mathrm{g}}(\mu, I)(\mathcal{A})=X-(X-\mathcal{A})_{g} \subseteq X-(X-\mathcal{B})_{g}=\Psi_{\mathrm{g}}(\mu, I)(\mathcal{B})$.

(3) One implication is immediate, i.e. $\Psi_{\mathrm{g}}(\mathcal{A} \cap \mathcal{B}) \subseteq \Psi_{\mathrm{g}}(\mathcal{A}) \cap \Psi_{\mathrm{g}}(\mathcal{B})$. Conversely, let $x \in \Psi_{\mathrm{g}}(\mathcal{A}) \cap \Psi_{\mathrm{g}}(\mathcal{B})$, then $x \in \Psi_{\mathrm{g}}(\mathcal{A})$ and $x \in \Psi_{\mathrm{g}}(\mathcal{B})$ from Definition 3.1, there exists $U_{1}, U_{2} \in N_{g}(x)$ such that $U_{1}-\mathcal{A} \in I$ and $U_{2}-\mathcal{B} \in I$. Let $U_{3}=U_{1} \cap U_{2}$ and we have $U_{3}-\mathcal{A} \in I$ and $U_{3}-\mathcal{B} \in I$ (by heredity of $I$ ), Thus $U_{3}-(\mathcal{A} \cap \mathcal{B})=\left(U_{3}-\mathcal{A}\right) \cup\left(U_{3}-\mathcal{B}\right) \in I$ (by additivity of $I$ ) and hence $x \in \Psi_{\mathrm{g}}(\mathcal{A} \cap \mathcal{B})$ which implies $\Psi_{\mathrm{g}}(\mathcal{A}) \cap \Psi_{\mathrm{g}}(\mathcal{B}) \subseteq \Psi_{\mathrm{g}}(\mathcal{A} \cap \mathcal{B})$ and this completes the proof.

(4) If $U \in \mu$, then $X-U$ is $\mu$-cloed set which implies $(X-U)_{g} \subseteq(X-U)$ (Theorem 2.8) and hence by Definition 3.1, we have $U \subseteq X-(X-U)_{g}=\Psi_{\mathrm{g}}(U)$.

(5) Since $\Psi_{\mathrm{g}}(\mathcal{A})$ is $\mu$-open set, then by (4) we have the result.

(6) Since $\Psi_{\mathrm{g}}(\mu, I)(\mathcal{A})=X-(X-\mathcal{A})_{g}$ and by hypothesis,

$$
\begin{aligned}
\Psi_{\mathrm{g}}\left(\Psi_{\mathrm{g}}(\mathcal{A})\right) & =\Psi_{\mathrm{g}}\left[X-(X-\mathcal{A})_{g}\right]=X-\left[X-\left(X-(X-\mathcal{A})_{g}\right)\right]_{g} \\
& =X-\left[(X-\mathcal{A})_{g}\right]_{g}=X-\left[(X-\mathcal{A})_{g}\right]_{g} \\
& =X-(X-\mathcal{A})_{g}=\Psi_{\mathrm{g}}(\mathcal{A})
\end{aligned}
$$

(7) Follows from Definition 3.1 and Theorem 2.1(9).

(8) From Theorem 2.1(9) and Definition 3.1, we have,

$$
\begin{aligned}
\Psi_{\mathrm{g}}(\mathcal{A}-\mathcal{E}) & =X-[X-(\mathcal{A}-\mathcal{E})]_{g}=X-[X-(\mathcal{A} \cap X-\mathcal{E})]_{g} \\
& =X-[(X-\mathcal{A}) \cup \mathcal{E}]_{g}=X-[(X-\mathcal{A})]_{g}=\Psi_{\mathrm{g}}(\mathcal{A})
\end{aligned}
$$

(9) From Theorem 2.1(9) and Definition 3.1, we have,

$$
\begin{aligned}
\Psi_{\mathrm{g}}(\mathcal{A} \cup \mathcal{E}) & =X-[X-(\mathcal{A} \cup \mathcal{E})]_{g}=X-[(X-\mathcal{A}) \cup \mathcal{E}]_{g} \\
& =X-[(X-\mathcal{A})]_{g}=\Psi_{\mathrm{g}}(\mathcal{A})
\end{aligned}
$$

(10) Follows immediately from (8) and (9).

Corollary 3.1. Let $(X, \mu, I)$ be an ideal generalized topological space for every $\mathcal{A} \subseteq X$ and $\mathcal{E} \in I$, then, $\Psi_{\mathrm{g}}(\mathcal{A}-\mathcal{E})=\Psi_{\mathrm{g}}(\mathcal{A})=\Psi_{\mathrm{g}}(\mathcal{A} \cup \mathcal{E})$.

Proof. Follows immediately from Theorem $3.2(8)$ and $3.2(9)$.

Theorem 3.3. Let $(X, \mu, I)$ be an ideal generalized topological space, then $\mu^{\star}=\left\{\mathcal{A} \subseteq X: \mathcal{A} \subseteq \Psi_{\mathrm{g}}(\mathcal{A})\right\}$. 
Proof. Let $\delta=\left\{\mathcal{A} \subseteq X: \mathcal{A} \subseteq \Psi_{\mathrm{g}}(\mathcal{A})\right\}$.

Firstly, we show that $\delta$ is a generalized topology. Observe that $\phi \subseteq \Psi_{\mathrm{g}}(\phi)$ and $\subseteq \Psi_{\mathrm{g}}(X)$. Now, if $\mathcal{A}, \mathcal{B} \subseteq \delta$, then by Theorem 3.2(3) we have $\mathcal{A} \cap \mathcal{B} \subseteq \Psi_{\mathrm{g}}(\mathcal{A} \cap \mathcal{B})=\Psi_{\mathrm{g}}(\mathcal{A}) \cap \Psi_{\mathrm{g}}(\mathcal{B})$, this implies that $\mathcal{A} \cap \mathcal{B} \in \delta$. If $\left\{\mathcal{A}_{i}\right\}_{i \in \Gamma} \subseteq \delta$, then $\mathcal{A}_{i} \subseteq \Psi_{\mathrm{g}}\left(\cup \mathcal{A}_{i}\right)$ for every $i \in \Gamma$. This implies $\cup \mathcal{A}_{i} \subseteq \Psi_{\mathrm{g}}\left(\cup \mathcal{A}_{i}\right)$ and we have shown that $\delta$ is a topology.

Secondly, we show that $\mu^{\star}=\delta$. Now if $U \in \mu^{\star}$ and $x \in U$, there exists $V \in \mu^{\star}$ and $\mathcal{E} \in I$ such that $x \in V-\mathcal{E} \subseteq U$. Clearly, $V-U \in \mathcal{E}$. so that $V-U \in I$ by heredity and hence $x \in \Psi_{\mathrm{g}}(U)$. Thus, $U \in \Psi_{\mathrm{g}}(U)$ and we have shown $\mu^{\star} \subseteq \delta$. Now, let $\mathcal{A} \in \delta$ we have by Definition 3.1 that $\mathcal{A} \in \Psi_{\mathrm{g}}(\mathcal{A})$, implies $\mathcal{A} \subseteq X-[(X-\mathcal{A})]_{g}$, which implies $(X-\mathcal{A})_{g} \subseteq(X-\mathcal{A})$, which also implies $X-\mathcal{A}$ is $\mu^{\star}$-open and hence $\mathcal{A} \in \mu^{\star}$. Thus, $\mu^{\star}=\delta$.

\section{Some Forms of $\star$-Compatible}

Definition 4.1. If $(X, \mu, I)$ is an ideal generalized topological space. Then I is said to be $\star$-compatible with $\mu$ denoted by $I \sim \mu$, if for every $\mathcal{A} \subseteq X$ and for every $x \in \mathcal{A}$, there exists $U \subseteq \mathcal{A}_{g}(x)$ such that $U \cap \mathcal{A} \in I$, then $\mathcal{A} \in I$.

Theorem 4.1. Let $(X, \mu, I)$ be an ideal generalized topological space. If $I \sim \mu$, then the base $\beta(\mu, I)$ for $\mu^{\star}(I)$ is a generalized topology and hence $\beta(\mu, I)=\mu^{\star}(I)$ and all $\mu$-open in $\mu^{\star}(I)$ are of simple form, i.e., $\mu^{\star}(I)=\{U-\mathcal{M}: U \in \mu\}$.

Proof. Follows immediately from the definition.

Theorem 4.2. If $I \sim \mu$, then the following statements are equivalent for a subset $\mathcal{A}$ of a space $(X, \mu, I)$.

(1) $\mathcal{A} \in I$.

(2) $\mathcal{A}_{g}=\phi$.

(3) $\mathcal{A} \cap \mathcal{A}_{g}=\phi$.

Proof. (1) $\Rightarrow(2)$ : Let $A \in I$. Then from hypothesis for every $x \in X$ and every $U \in N_{g}(x)$, we have $U \cap \mathcal{A} \in I$. Thus $x \notin \mathcal{A}_{g}$ which means $\mathcal{A}_{g}=\phi$.

$(2) \Rightarrow(3)$ : Obvious.

$(3) \Rightarrow(1): \mathcal{A} \notin I$. Then according to $\star$-compatible of $\mu$ with $I$, there is an $x \in \mathcal{A}$ such that for every $U \in N_{g}(x), U \cap \mathcal{A} \notin I$, so $x \in \mathcal{A}_{g}$ which means $\mathcal{A} \cap \mathcal{A}_{g} \neq \phi$, which is a contradiction. 
Theorem 4.3. Let $(X, \mu, I)$ be an ideal generalized topological space. Then $I \sim \mu$. iff $\Psi_{\mathrm{g}}(\mathcal{A})-\mathcal{A} \in I$, for every $\mathcal{A} \subseteq X$.

Proof. Necessity Let $I \sim \mu$ and $\mathcal{A} \subseteq X$. We observe that $x \in \Psi_{\mathrm{g}}(\mathcal{A})-\mathcal{A}$ iff $x \notin \mathcal{A}$ and $x \notin(X-\mathcal{A})_{g}$ iff $x \notin \mathcal{A}$ and there exists $U \in N_{g}(x)$, such that $U-\mathcal{A} \in I$, iff there exists $x \in U \in N_{g}(x)$, such that $x \in(U-\mathcal{A}) \in I$. Now for each $x \in \Psi_{\mathrm{g}}(\mathcal{A})-\mathcal{A}$ and $U \in N_{g}(x)$ and $U \cap\left(\Psi_{\mathrm{g}}(\mathcal{A})-\mathcal{A}\right) \in I$, by heredity, since $I \sim \mu$, then $\left(\Psi_{\mathrm{g}}(\mathcal{A})-\mathcal{A}\right) \in I$.

Sufficiency: Let $\mathcal{A} \subseteq X$, and let for every $x \in \mathcal{A}$ there exists $U \in N_{g}(x)$ such that $(U \cap \mathcal{A}) \in I$ and now we prove that $\mathcal{A} \in I$. We observe that $\Psi_{\mathrm{g}}(X-\mathcal{A})-(X-\mathcal{A})=\left\{x: \exists U \in N_{g}(x)\right.$ such that $x \in\{[U-(X-\mathcal{A})] \in I\}\}$. This implies $\Psi_{\mathrm{g}}(X-\mathcal{A})-(X-\mathcal{A})=\left\{x: \exists U \in N_{g}(x)\right.$ such that $\left.x \in(U \cap \mathcal{A}) \in I\right\}$. Thus we have $\mathcal{A} \subseteq \Psi_{\mathrm{g}}(X-\mathcal{A})-(X-\mathcal{A}) \in I$ and hence $\mathcal{A} \in I$ by the heredity of $I$.

Theorem 4.4. Let $(X, \mu, I)$ be an ideal generalized topological space with $I \sim \mu$. Then

$(1)\left(\mathcal{A}-\mathcal{A}_{g}\right) \in I$, for every $\mathcal{A} \subseteq X$.

(2) $\left(\mathcal{A}_{g}\right)_{g}=\mathcal{A}_{g}$, for every $\mathcal{A} \subseteq X$.

Proof. Assume that $x \in(\mathcal{A}-\mathcal{A})_{g}$, then for every $U \in N_{g}(x)$, we have $\left(\mathcal{A}-\mathcal{A}_{g}\right) \cap U \notin I$ or $(U \cap \mathcal{A})-\mathcal{A}_{g} \notin I$. This implies $(\mathcal{A} \cap U) \notin I$ and so $x \in \mathcal{A}_{g}$. Thus, $x \in \mathcal{A}-\mathcal{A}_{g}$ from which we conclude that $\left(\mathcal{A}-\mathcal{A}_{g}\right) \cap\left(\mathcal{A}-\mathcal{A}_{g}\right)_{g}=\phi$, from Theorem 4.2 we have $\mathcal{A}-\mathcal{A}_{g} \in I$.

(2) Let $I \sim \mu$. Then for every $\mathcal{A} \subseteq X, \mathcal{A}-\mathcal{A}_{g} \in I$. This implies $\mathcal{A}-\mathcal{A}_{g}=\phi$, by using Theorem 2.1(7), we have $\mathcal{A}_{g}-\left(\mathcal{A}_{g}\right)_{g} \subseteq\left(\mathcal{A}-\mathcal{A}_{g}\right)_{g}=\phi$. Hence, $\mathcal{A}_{g} \subseteq\left(\mathcal{A}_{g}\right)_{g}$. However, by Theorem 2.1(4) we have $\mathcal{A}_{g} \subseteq\left(\mathcal{A}_{g}\right)_{g}$. Thus, $\mathcal{A}_{g}=\left(\mathcal{A}_{g}\right)_{g}$.

Theorem 4.5. Let $(X, \mu, I)$ be an ideal generalized topological space with $I \sim \mu$. Then $\Psi_{\mathrm{g}}\left(\Psi_{\mathrm{g}}(\mathcal{A})\right)=\Psi_{\mathrm{g}}(\mathcal{A})$ for every $\mathcal{A} \subseteq X$.

Proof. From Theorem 3.2(5) we have $\Psi_{\mathrm{g}}(\mathcal{A}) \subseteq \Psi_{\mathrm{g}}\left(\Psi_{\mathrm{g}}(\mathcal{A})\right)$. Since $I \sim \mu$, then from Corollary 3.1, $\Psi_{\mathrm{g}}(\mathcal{A})=$ $\mathcal{A} \cup \mathcal{E}$ for some $\mathcal{E} \in I$ and hence by Theorem 3.2(9) $\Psi_{\mathrm{g}}\left(\Psi_{\mathrm{g}}(\mathcal{A})\right)=\Psi_{\mathrm{g}}(\mathcal{A} \cup \mathcal{E})=\Psi_{\mathrm{g}}(\mathcal{A})$.

Acknowledgement: The author would like to express their sincere thanks to the referees for their useful suggestions and comments.

Conflicts of Interest: The author declares that there are no conflicts of interest regarding the publication of this paper. 


\section{REFERENCES}

[1] A. Császár, Generalized topology, generalized continuity, Acta Math. Hungar. 96 (2002), 351-357.

[2] A. Császár, Generalized open sets in generalized topologies, Acta Math. Hun gar. 106 (12) (2005), 53-66.

[3] A. Császár, Further remarks on the formula for -interior, Acta Math. Hungar. 113 (2006), 325-332.

[4] A. Császár, Modification of generalized topologies via hereditary classes, Acta Math. Hungar. 115 (2007), 29-36.

[5] A. Al-Omari and T. Noiri, Local closure functions in ideal topological spaces, Novi Sad J. Math. 43(2) (2013), 139-149.

[6] M. Caldas, S. Jafari and R.M. Latif, Sobriety via $\theta$-open sets, An. S, tiint,. Univ. Al. I. Cuza Ias,i. Mat. (N.S.), 56(1) (2010), 163-167.

[7] F. Cammaroto, A. Catalioto, B.A. Pansera and B. Tsaban, On the cardinality of the $\theta$-closed hull of sets, Topol. Appl. 160(18) (2013), 2371-2378.

[8] R.F. Dickman Jr and J.R. Porter, $\theta$-closed subsets of Hausdorff spaces, Pac. J. Math. 59(2) (1975), 407-415.

[9] S. Fomin, Extensions of topological spaces, Ann. Math. 44 (3) (1943), 471-480.

[10] G. Freud, Ein Beitrag zu dem Satze von Cantor und Bendixson, Acta Math. Acad. Sci. Hungar. 9 (1958), $333-336$.

[11] S. Jafari and N. Rajesh, Generalized closed sets with respect to an ideal, Eur. J. Pure Appl. Math. 4(2) (2011), $147-151$.

[12] D.S. Janković, On some separation axioms and $\theta$-closure, Mat. Vesnik, 4 (17) (32) (72) (1980), 439-450.

[13] D. Janković and T.R. Hamlett, New topologies from old via ideals. Amer. Math. Mon. 97(4) (1990), 295-310.

[14] K. Kuratowski, Topologie I, Warszawa, 1933.

[15] K. Kuratowski, Topology, Vol. I, Academic Press, New York, London, 1966.

[16] R. Manoharan and P. Thangavelu, Some new sets and topologies in ideal topological spaces, Chin. J. Math. 2013 (2013), Article ID 973608.

[17] S. Scheinberg, Topologies which generate a complete measure algebra, Adv. Math. 7 (1971), 231-239.

[18] R. Vaidyanathaswamy, The localisation theory in set-topology. Proc. Indian Acad. Sci., Sect. A. 20 (1944), 51-61.

[19] N.V. Velicko, H-closed topological spaces. Mat. Sb. (N.S.) 70 (112) (1966) 98-112 (in Russian); in: American Mathematical Society Translations, vol. 78, American Mathematical Society, Providence, RI, (1969), 103-118

[20] F. Alsharari and Y. M. Saber, $\mathcal{G} \Theta_{\tau_{i}}^{\star \tau_{j}}$-Fuzzy closure operator, New Math. Nat. Comput. in press. https://doi.org/10.1142/S1793005720500088

[21] A.M. Zahran, S.E. Abbas, S.A. Abd El-baki and Y.M. Saber, Decomposition of fuzzy continuity and fuzzy ideal continuity via fuzzy idealization, Chaos Solitons Fractals, 42 (2009), 3064-3077.

[22] Y.M. Saber and M.A. Abdel-Sattar, Ideals on Fuzzy Topological Spaces, Appl. Math. Sci. 8 (2014), 1667 - 1691.

[23] Y.M. Saber and F. Alsharari, Generalized Fuzzy Ideal Closed Sets on Fuzzy Topological Spaces in $\breve{S}$ ostak Sense, Int. J. Fuzzy Logic Intell. Syst. 18 (3) (2018), 161-166.

[24] F. Alsharari and Y.M. Saber, Separation axioms on fuzzy ideal topological spaces in $\breve{S}$ ostak's sense, Int. J. Adv. Appl. Sci. 7 (2) (2020), $78-84$.

[25] Y.M. Saber, F. Alsharari and F. Smarandache, On Single-Valued Neutrosophic Ideals in $\breve{S}$ ostak Sense, Symmetry, 12 (2) (2020), 193. 